\title{
Papel do Fisioterapeuta em diferentes cenários de atuação à COVID-19*
}

\author{
The role of physiotherapy in different COVID-19 scenarios
}

\author{
MARTINEZ, Bruno Prata'; ANDRADE, Flávio Maciel Dias de²; MARTINS, Jocimar \\ Avelar ${ }^{3}$; MATTE, Darlan Lauríció; KARSTEN, Marlus ${ }^{5}$, em nome do Comitê COVID-19 da \\ ASSOBRAFIR.
}

\begin{abstract}
Resumo
Este documento fornece orientação sobre o papel do fisioterapeuta em diferentes cenários para lidar com a COVID-19. Desde dezembro de 2019, com o surgimento do primeiro caso de COVID-19 e a expansão de casos em todo o mundo, muitos esforços foram direcionados para conter a pandemia. O fisioterapeuta, como um membro importante da equipe de saúde, desempenha um papel importante em diferentes cenários de desempenho, do primário ao terciário. Este documento apresenta uma tabela com os cenários e ações a serem realizados no nível primário, antes de uma possível contaminação, bem como as estratégias de tratamento no nível hospitalar, antes e após a intubação orotraqueal.
\end{abstract}

Palavras-chave: Fisioterapia; prevenção primária; COVID-19.

\footnotetext{
Revisado por membros do Comitê COVID-19 da ASSOBRAFIR, nomeado por meio do memorando No003/2020. Esta publicação é uma atualização da Comunicação Oficial "Papel do Fisioterapeuta em diferentes cenários de atuação à COVID-19", chancelada pelo Comitê COVID-19 da ASSOBRAFIR, originalmente escrita pelos mesmos autores e divulgada em 25/03/2020 no endereço eletrônico https://assobrafir.com.br/covid-19-papel-do-fisioterapeuta-emdiferentes-cenarios-de-atuacao.

1 Programa de Pós-Graduação em Medicina e Saúde, Universidade Federal da Bahia(UFBA), Salvador, Bahia, Brasil. Colegiado de Fisioterapia, Universidade do Estado da Bahia(UNEB), Salvador, Bahia, Brasil. E-mail: brunopmartinez@ hotmail.com. BPM - https://orcid.org/ 0000-0002-4673-8698

2 Universidade Católica de Pernambuco, Recife, Pernambuco, Brasil. FMDA - https://orcid.org/0000-0001-9571-6551

${ }_{3}$ Faculdade Dinâmica do Vale do Piranga, Ponte Nova, Minas Gerais, Brasil. JAM - https://orcid.org/0000-0002-5023-3131

${ }_{4}^{4}$ Programa de Pós-Graduação em Fisioterapia, Universidade do Estado de Santa Catarina (UDESC), Florianópolis, Santa Catarina, Brasil. Programa de Pós-Graduação em Ciências do Movimento Humano, UDESC, Florianópolis, Santa Catarina, Brasil. Departamento de Fisioterapia, UDESC, Florianópolis, Santa Catarina, Brasil.

DLM - https://orcid.org/0000-0003-4650-3714

${ }_{5}$ Programa de Pós-Graduação em Fisioterapia, Universidade do Estado de Santa Catarina (UDESC), Florianópolis, Santa Catarina, Brasil. Departamento de Fisioterapia, UDESC, Florianópolis, Santa Catarina, Brasil. Programa de PósGraduação em Ciências da Reabilitação, Universidade Federal de Ciências da Saúde de Porto Alegre (UFCSPA), Porto Alegre, Rio Grande do Sul, Brasil. MK - https://orcid.org/0000-0002-2476-7981
} 


\section{Abstract}

This document provides guidance on the role of physiotherapy in different COVID-19 scenarios. Since December 2019 when the first case of COVID-19 was reported, followed by the rapid increase in the number of cases around the world many efforts have been directed to contain the pandemic. The physiotherapist as an important member of the healthcare team plays an important role treating patients with COVID-19 in different settings - from primary to tertiary care. The present article provides a chart with actions to be performed at the primary level, before the infection by the disease, as well as treatment strategies at the hospital level before and after endotracheal intubation.

Keywords: Physiotherapy; primary prevention; COVID-19.

\section{Objetivo}

O objetivo do presente posicionamento é fornecer orientações resumidas sobre o papel do fisioterapeuta em diferentes cenários no enfrentamento da COVID-19 de forma simplificada através de um quadro ilustrativo.

\section{Contextualização}

O presente quadro ilustra algumas orientações com possíveis ações do fisioterapeuta dentro do combate a pandemia pela COVID-19, desde a atenção primária, para não ocorrência de novos casos, até a assistência no ambiente hospitalar para tratamento da insuficiência respiratória desencadeada nos quadros mais graves.

A nível de atenção básica, a principal estratégia para não ocorrência de novos infectados pelo vírus Sars-Cov2 é o distanciamento social; já que este evitará a transmissão, a qual ocorre pelo contato do vírus com as mucosas. Assim, cabe ao fisioterapeuta o reforço das orientações para população a nível da atenção básica para que haja o possível achatamento da curva, prevenindo assim a falta de leitos necessários para pessoas com quadros graves.

Nos pacientes com os sintomas da COVID-19, associado a falta de ar, frequência respiratória maior que 24 incursões por minuto e/ou oxigenação reduzida $\left(\mathrm{SpO}_{2}<93 \%\right)$ é necessário a ida a um hospital para avaliação diagnóstica e início dos tratamentos necessários. É importante que esses parâmetros sejam respeitados na ida ao hospital, para que o cidadão não vá ao hospital, sem que haja uma necessidade efetiva, expondo-o assim a um maior risco de adquirir a COVID-19, ao longo deste percurso, recomenda-se a observação dos sintomas para esta tomada de decisão.

Após a internação hospitalar quando indicada, algumas estratégias e intervenções serão iniciadas conforme necessidade, as quais estão descritas no quadro e são abordadas em maior profundidade nos posicionamentos emitidos pelo Comitê COVID-19 da ASSOBRAFIR. 

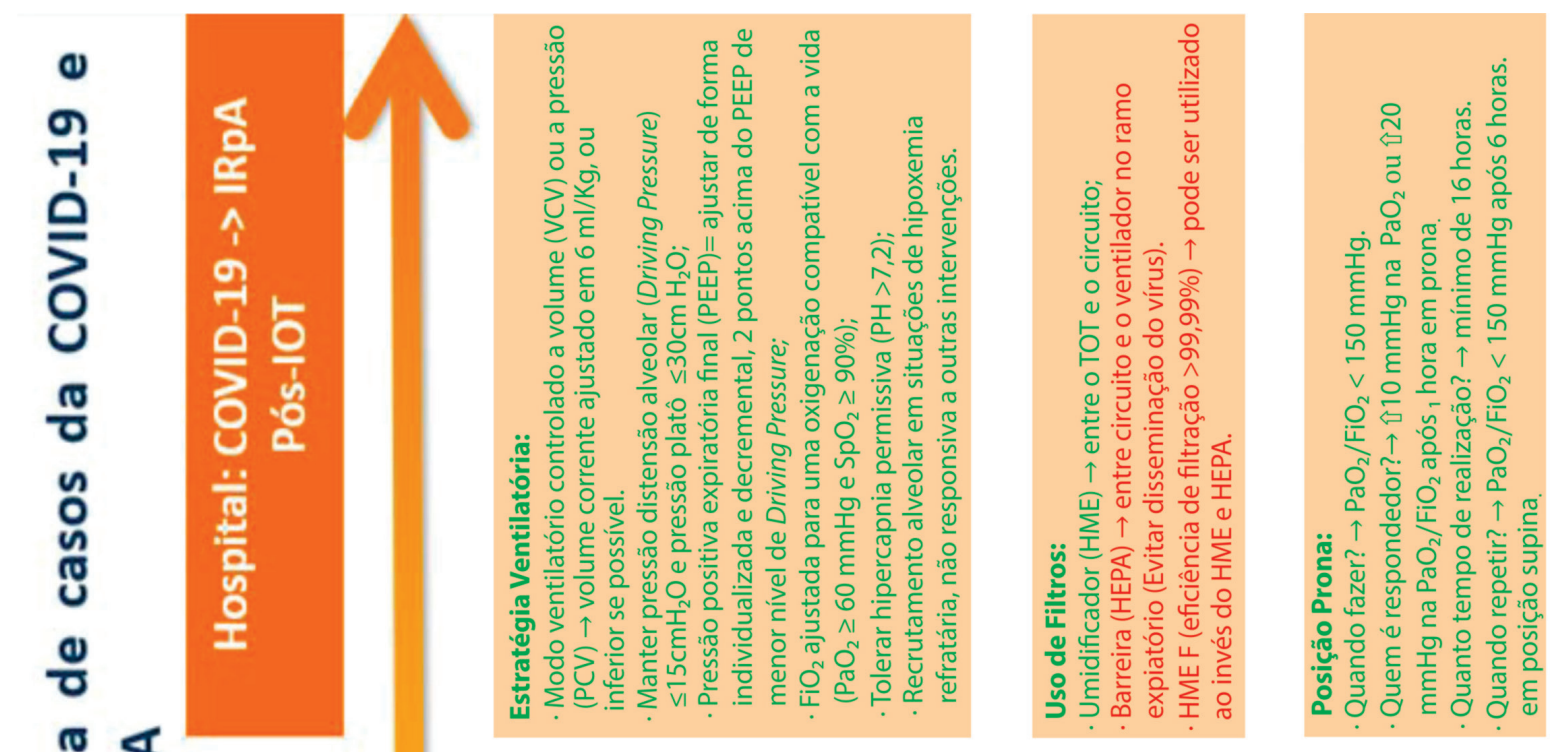

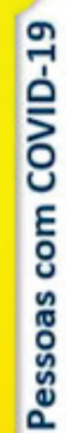
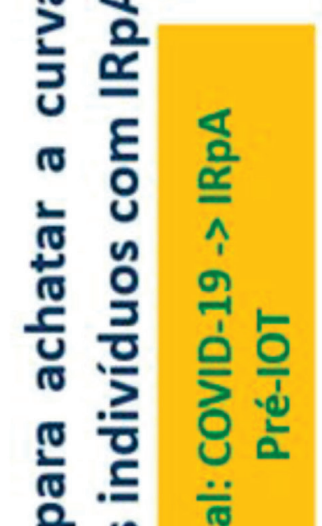

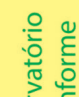
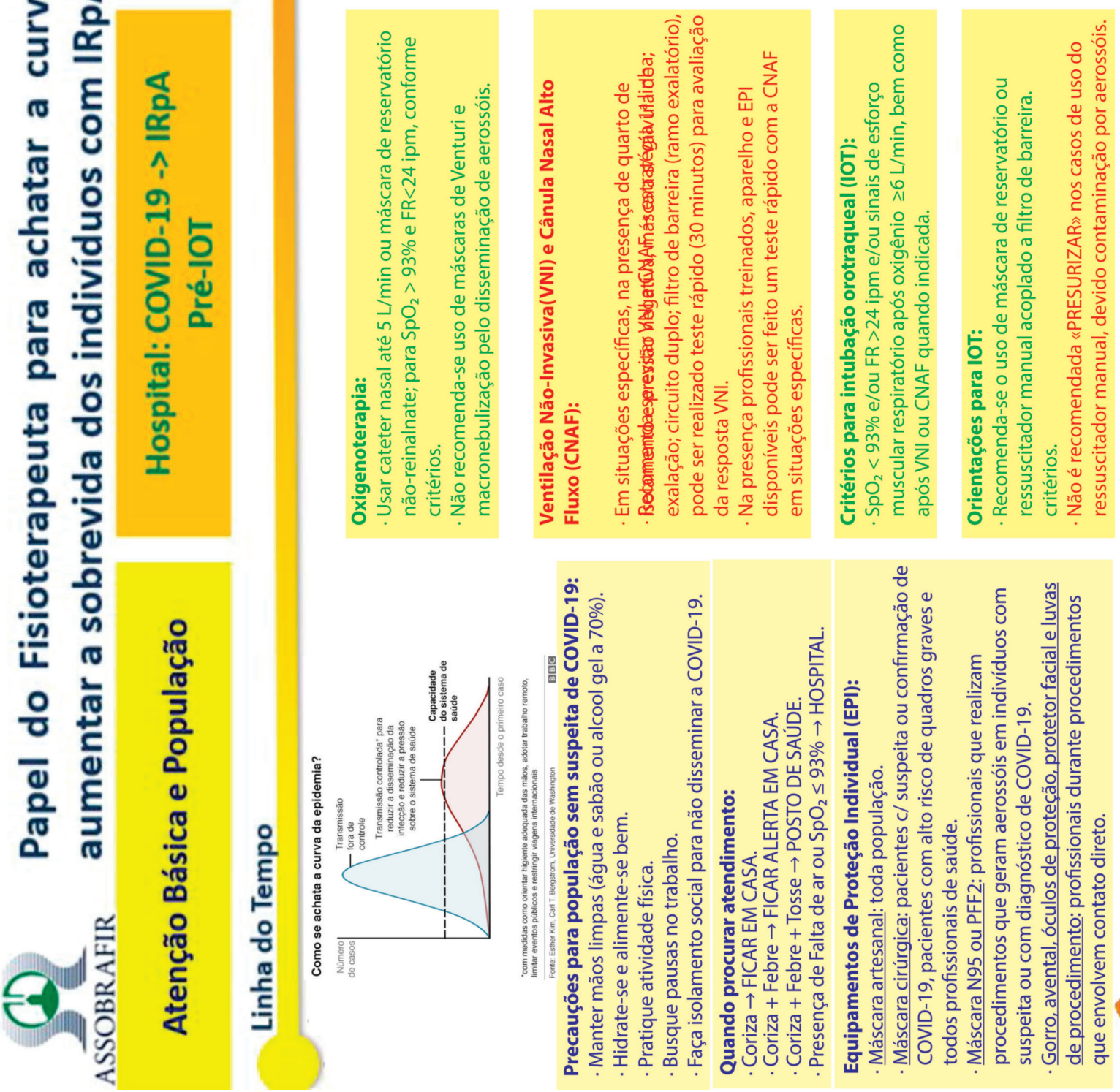
近岁全言

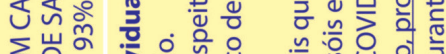

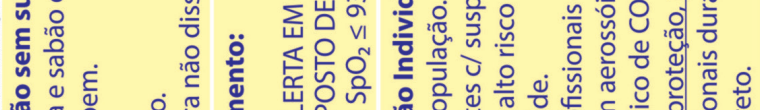

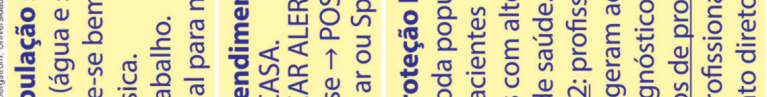

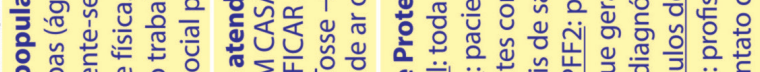

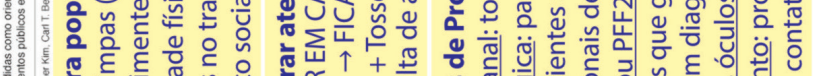

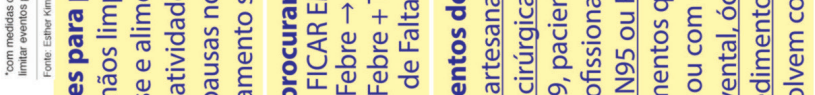

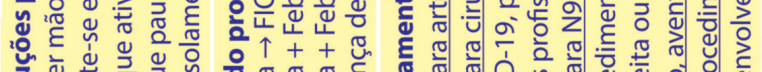

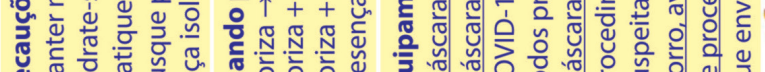

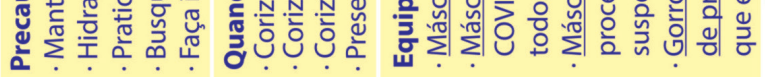




\section{Referências}

1. World Health Organization. Clinical management of severe acute respiratory infection (SARI) when COVID-19 disease is suspected: interim guidance, 13 March 2020. Geneva; World Health Organization; 2020.

2. Ministério da Saúde, Secretaria de Atenção Especializada à Saúde, Departamento de Atenção Hospitalar, Domiciliar e de Urgência. Protocolo de manejo clínico da Covid-19 na Atenção Especializada [Internet]. 1. ed. rev. - Brasília: Ministério da Saúde; 2020. Available from: http://bvsms.saude.gov.br/ bvs/publicacoes/manejo clinico covid-19 atencao especializada.pdf

3. Marini JJ, Gattinoni L. Management of COVID-19 Respiratory Distress. JAMA. 2020;323(22):23292330. Published online April 24, 2020. doi:10.1001/jama.2020.6825

4. Alhazzani W, Møller MH, Arabi YM, Loeb M, Gong MN, Fan E, et al. Surviving Sepsis Campaign: Guidelines on the Management of Critically Ill Adults With Coronavirus Disease 2019 (COVID-19). Crit Care Med . 2020 Jun;48(6):e440-e469.

5. Alhazzani W, Møller MH, Arabi YM, Loeb M, Gong MN, Fan E, et al. Surviving Sepsis Campaign: Guidelines on the Management of Critically Ill Adults With Coronavirus Disease 2019 (COVID-19). Intensive Care Med. 2020 May;46(5):854-887.

6. Guérin C, Reignier J, Richard J, Beuret P, Gacouin A, Boulain T, et al. Prone Positioning in Severe Acute Respiratory Distress Syndrom. N Engl J Med 2013;368:2159-2168. doi: 10.1056/NEJMoa1214103.

7. AMIB. Orientações sobre o manuseio do paciente com pneumonia e insuficiência respiratória devido a infecção pelo coronavírus (SARS-CoV-2) - Versão n.05/2020 [Internet]. São Paulo: Associação de Medicina Intensiva Brasileira; 2020. Available from: https://www.amib.org.br/fileadmin/user upload/amib/2020/ abril/24/vm ai vjs v5 abr 2020 final.pdf

8. Grass Elli G, Zangrillo A, Zanella A, Antonelli M; Cabrini L; Castelli A, et al. Baseline Characteristics and Outcomes of 1591 Patients Infected With SARS-CoV2 Admitted to ICU sof the Lombardy Region, Italy. JAMA. 2020;323(16):1574-1581. Published online 2020 Apr 6. doi:10.1001/jama.2020.5394.

9. Yang X, Yu Y, Xu J, Shu H, Xia J, Liu H, et al. Clinical course and outcomes of critically ill patients with SARS-CoV-2 pneumonia in Wuhan, China: a single-centered, retrospective, observational study. Lancet Respir Med. 2020 May; 8(5): 475-481. Published online 2020 Feb 24. doi: 10.1016/S22132600(20)30079-5

10. Wang K, Zhao W, Li J, Shu W, Duan J. The experience of high-flow nasal cannula in hospitalized patients with 2019 novel coronavirus-infected pneumonia in two hospitals of Chongqing, China. Ann Intensive Care. 2020; 10: 37. Published online 2020 Mar 30. doi: 10.1186/s13613-020-00653-Z

Submissão em: 08/06/2020

Aceite em: 15/07/2020 